\title{
Retraction Note to: Evaluation of the pulley function of the hamulus of the hamate for the flexor tendons
}

\author{
Kenji Goto $^{1}$ Kiyohito Naito ${ }^{1}$ Yoichi Sugiyama ${ }^{1}$ Mayuko Kinoshita ${ }^{1} \cdot$ Nana Nagura $^{1,2} \cdot$ Yoshiyuki Iwase $^{2}$. \\ Kazuo Kaneko'
}

Published online: 4 October 2021

(c) Springer-Verlag France SAS, part of Springer Nature 2021

\section{Retraction to: \\ Surgical and Radiologic Anatomy (2018) 40:1013-1017 https://doi.org/10.1007/s00276-018-2034-0}

Publisher's Note Springer Nature remains neutral with regard to jurisdictional claims in published maps and institutional affiliations.

The authors have retracted this article because they did not obtain ethics approval prior to commencing the study. All authors agree to this retraction.

The original article can be found online at https://doi.org/10.1007/ s00276-018-2034-0.

Kiyohito Naito

knaito@juntendo.ac.jp

1 Department of Orthopaedics, Juntendo University School of Medicine, Juntendo University, 2-1-1 Hongo, Bunkyo-ku, Tokyo 113-8421, Japan

2 Department of Orthopaedic Surgery, Juntendo Tokyo Koto Geriatric Medical Center, 3-3-20 Shinsuna, Koto-ku, Tokyo 136-0075, Japan 